\title{
UV photon-counting CCD detectors that enable the next generation of UV spectroscopy missions: AR coatings that can achieve $80-90 \%$ QE
}

\author{
Erika T. Hamden ${ }^{a}$, Frank Greer ${ }^{b}$, David Schiminovich ${ }^{a}$, Shouleh Nikzad $^{b}$, \\ D. Christopher Martin ${ }^{c}$ \\ ${ }^{a}$ Department of Astronomy, Columbia University, 550 W 120th St, New York, NY 10025, USA \\ ${ }^{b}$ Jet Propulsion Laboratory, California Institute of Technology, M/S 302-304, Pasadena, CA \\ 91109, USA ${ }^{c}$ Department of Astronomy, California Institute of Technology, 1200 East \\ California Blvd, Pasadena, CA 91125, USA
}

\begin{abstract}
We describe recent progress in the development of anti-reflection coatings for use at UV wavelengths on CCDs and other Si-based detectors. We have previously demonstrated a set of coatings which are able to achieve greater than $50 \%$ QE in 4 bands from $130 \mathrm{~nm}$ to greater than $300 \mathrm{~nm}$. We now present new refinements of these AR-coatings which will improve performance in a narrower bandpass by $50 \%$ over previous work. Successful test films have been made to optimize transmission at 190nm, reaching $80 \%$ potential transmission.
\end{abstract}

Keywords: UV, Anti-reflection coatings, thin-films, CCDs

\section{INTRODUCTION}

Anti-reflection (AR) coatings have been used in a wide range of applications to increase transmission through a surface, including on CCDs at visible wavelengths. While CCDs have traditionally not been effective in the $\mathrm{UV}$, treatments have been developed solve this problem. Backside illumination and Delta Doping can bring the quantum efficiency $(\mathrm{QE})$ of treated devices up to the reflection limit of silicon at all wavelengths in a stable and consistent way; ${ }^{1}$ in the UV this brings the QE to around $30 \%$. This non-zero QE can then be increased to a much higher value with the selection of a good AR coating. Our initial work on single layer AR coatings at UV wavelengths has been successful at increasing QE to greater than $50 \%$ from $135 \mathrm{~nm}$ and longer ${ }^{2}{ }^{3}$ While these coatings are a good start, they are indicative of what is possible with more complex designs.

The use of multi-layer AR-coatings at visible and IR wavelengths can provide near $100 \%$ transmission. Reaching that at UV wavelengths is a challenge due to the limited number of non-absorbing materials that can be used to make coatings. While adding a multi-layer coating to any surface can change the transmission through it, there are preferred configurations of thickness and index which will minimize reflectance. ${ }^{4}$ In this discussion, we restrict our work to those configurations dealing with high-index substrates, such as silicon, since these coatings are all designed for use on silicon CCDs. The first method to create AR coatings pairs a high and low index material, where both indices lie between the index of the substrate and 1.0, the index of air. Multiple layers (typically based on quarter wave thicknesses or multiples thereof) which alternate between the materials combine to minimize reflectance to nearly zero at a selected wavelength and usually twice that wavelength. Adjustment can be made to optimize the range of zero reflectance and to refine the film for best outcome. Here we do this using a computer program such as TFCalc. The quarter wave stack method works best when the index of refraction of the materials used (including the substrate) are not varying significantly over the wavelength range in question. Other limitations include the lack of a low index material lower than $1.37\left(\mathrm{MgF}_{2}\right)$.

A second method is to slowly vary the index of refraction from the substrate to air. This method uses layers of material that step down the index, all approximately a quarter wave thick. Films with highest index lie closest to the substrate, with each subsequent film having a lower index. Depending on the substrates and materials

Corresponding Author: E-mail hamden@astro.columbia.edu

High Energy, Optical, and Infrared Detectors for Astronomy V, edited by Andrew D. Holland, James W. Beletic, Proc. of SPIE Vol. 8453, 845309 - (c) 2012 SPIE · CCC code: 0277-786X/12/\$18 · doi: 10.1117/12.927208 
involved, these films will be optimized for large bandpasses and so can be an important component of imagers, which often require sensitivity over hundreds of nanometers. Typically, these broadband designs are made at wavelengths with a constant index of refraction for the substrate and layer materials. Silicon at UV wavelengths (100-300nm) has extremely variable optical constants, and so high QE, very broadband coatings like those made for optical wavelengths are not easily created. Instead, what we focus on in this paper is high QE at the expense of broad application and discuss only narrowband coatings for specific applications, made using multiple layers of high and low index material.

Lastly, there are more innovative AR-coatings which rely on 3-d nano structures to construct a pseudo-moth's eye film. These structures, often pyramids or cone shapes made out of the same material as the underlying substrate, are smaller than the wavelength of light observed. Their size and tapered shape cause a gradual change in the index of refraction from air to substrate in a smooth way. The light never encounters a "surface" to refract/reflect off, and so there is very little reflection. New techniques have attempted to apply this concept down to the NUV, but the cone size required is so small as to pose quite a challenge. ${ }^{5}$

Here we focus on multi-layer AR-coatings which are optimized for several UV wavelengths. Our first test multi-layers have focused on models centered near $200 \mathrm{~nm}$. This wavelength was selected since it is the location of an atmospheric window between $\mathrm{O}_{2}$ and $\mathrm{O}_{3}$ absorption bands, ${ }^{6}$ which allows for observation in the UV at balloon and rocket altitudes. A balloon experiment, the Faint Intergalactic Redshifted Experiment Balloon (FIREBall), ${ }^{7}$ is just such a mission. This balloon experiment is funded for a third launch, and is an ideal test bed for a delta-doped AR-coated CCD. With this in mind, we have selected several models as first tests.

In Section 2, we describe techniques for model creation and the models themselves. In Section 3, we describe deposition recipes and techniques, along with growth rates for each material. In Section 4, we describe the reflectance and transmission measurements of the films on inert substrates.

\section{MODEL DEVELOPMENT}

Multi-layer films were modeled using TFcalc. Materials under consideration include $\mathrm{MgO}, \mathrm{MgF}_{2}, \mathrm{Al}_{2} \mathrm{O}_{3}, \mathrm{SiO}_{2}$, and $\mathrm{HfO}_{2}$. For uses below 220nm, we eliminate $\mathrm{HfO}_{2}$ as a viable film layer due to absorption. Below 200nm, we eliminate $\mathrm{MgO}$, and below 180 we eliminate $\mathrm{Al}_{2} \mathrm{O}_{3}$. For the shortest wavelength applications, multi-layers can be made of $\mathrm{MgF}_{2}$ and $\mathrm{SiO}_{2}$. The use of alternative fluorides, such as $\mathrm{CaF}_{2}$ and $\mathrm{LaF}_{3}$, can increase the options for high quality coatings at extreme UV wavelengths, although those options are not explored here. Optical constants come from Palik ${ }^{8},{ }^{9}$ although in some cases constants used are from samples made at JPL and measured by J.A. Woollam using vacuum ellipsometry.

In previous work, we have used standard silicon optical constants to determine the expected reflectance and transmittance of film materials. However, further study has found that the optical constants of delta-doped $\mathrm{Si}$ are slightly different than undoped Si. Alternative optical constants for delta-doped Si were measured by the J.A. Woollam. While the difference is slight at most wavelengths, the reflectance can change by several percent at UV wavelengths, and so we include this layer in our calculations. As such, we use the following structure in our reflectance calculations: a bulk Si substrate, a 3.413nm thick delta-doped Si layer with alternative constants, and a $1.7 \mathrm{~nm}$ thick native $\mathrm{SiO}_{2}$ oxide. In this calculation, we determine the reflectance off of the stack and any added coatings. Absorption is not a concern for the delta doped layer, as any electrons created there are still detected in the CCD. Absorption calculations take into account the native oxide and any added coatings only. Anticipated transmission is calculated by subtracting reflectance and absorption from $100 \%$.

We have developed a suite of sample films to increase QE above the reflectance limit of $\mathrm{Si}$, in some cases reaching $90 \%$, at a few test wavelengths. We present plots of expected transmission for films optimized at $155 \mathrm{~nm}$, 205nm (Figure 1), 255nm (Figure 2), and 305nm (Figure 3).

The most difficult range is certainly $155 \mathrm{~nm}$, where very few materials are available to create multi-layer coatings. Additionally, due to the limited range of optical constants, the maximum QE achievable is not much greater than that achieved with a single layer. At longer wavelengths, such as 205 and 255, there are more materials available and correspondingly more appropriate constants. Potential QE well above $80 \%$ is achievable with a few layers. Finally, films made above $300 \mathrm{~nm}$ can achieve near perfect transmission, although truly wide 

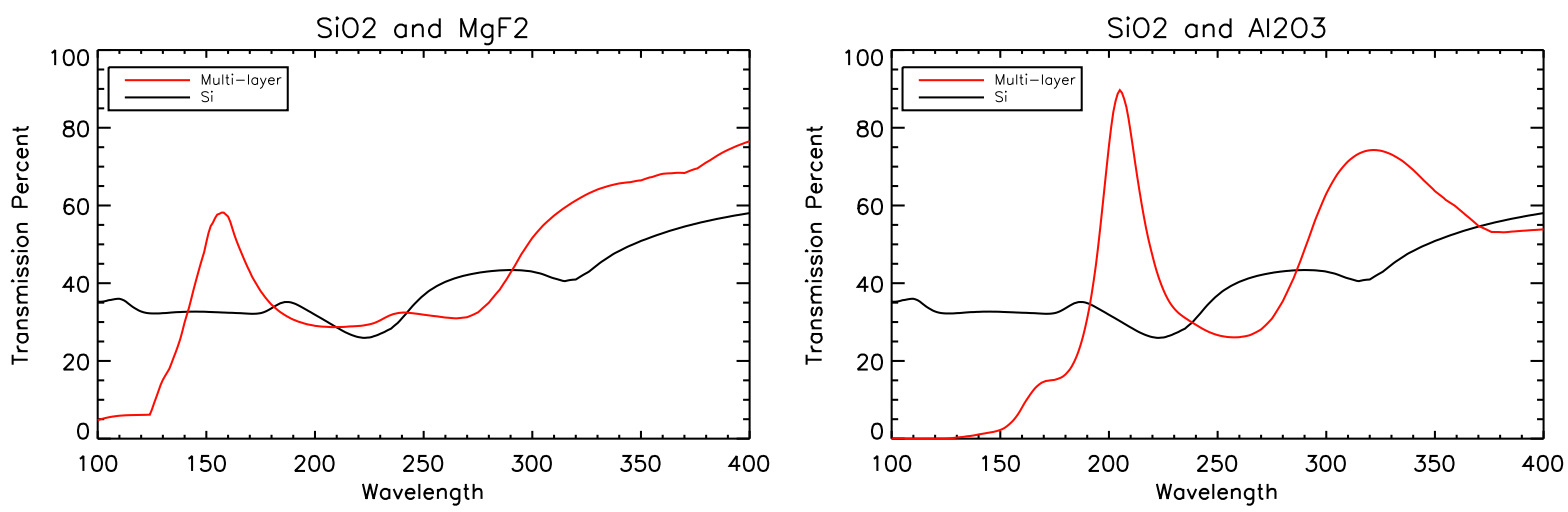

Figure 1. At left: Expected transmission for a multilayer model optimized for maximum transmission at $155 \mathrm{~nm}$ using $\mathrm{SiO}_{2}$ and $\mathrm{MgF}_{2}$ as materials. At right: Expected transmission for a multilayer model optimized for maximum transmission at 205nm using $\mathrm{SiO}_{2}$ and $\mathrm{Al}_{2} \mathrm{O}_{3}$ as materials.

bandpasses (hundreds of $\mathrm{nm}$ ) with high QE (above $90 \%$ for the whole range) do not become possible until above $370 \mathrm{~nm}$.

Finally, we summarize each model in Table 1. Listed characteristics are peak QE and at what wavelength, materials, and width of band at 50\% QE. Typical FWHM for bandpasses are less useful here since the out of band QE is not zero, but typically closer to the reflectance limit of silicon. Therefore, we will just use the bandwidth at $50 \%$ to set a standard for comparison between film models in this paper.

\section{DEPOSITION TECHNIQUES}

All depositions were made using Atomic Layer Deposition (ALD) at NASA's Jet Propulsion Laboratory. ALD enables conformal, atomic-layer level control over film growth and allows well controlled, repeatable depositions. ALD recipes for $\mathrm{Al}_{2} \mathrm{O}_{3}$ came from Goldstein, ${ }^{10} \mathrm{MgO}$ from a custom reaction developed at JPL by the authors, $\mathrm{SiO}_{2}$ from Dingemans ${ }^{12}$ and $\mathrm{HfO}_{2}$ from Liu. ${ }^{13}$ Often growth recipes required modifications to accommodate the equipment used. Modified growth recipes were typically validated using either XPS, TEM, or both. Growths were typically conducted at $200^{\circ} \mathrm{C}$.

Films were grown on 1 inch $<100>1-20 \mathrm{Ohm}-\mathrm{cm}$ silicon wafers (to test reflectance) and on fused silica windows (to test absorption). As noted above, differing optical constants between un-doped and doped Si means the films tested here are slight modifications to the ones described above. All measurements (and corresponding models) plotted are based on a silicon substrate without the doped layer. We are still able to test the overall nature of the film, its behavior compared to modeled on normal silicon, and we expect few changes when grown

Table 1. Table summarizing characteristics of each film model pictured.

\begin{tabular}{|c|c|c|c|c|}
\hline Model & Materials & Max QE $\%$ & $\lambda$ of Max $(\mathrm{nm})$ & Width at 50\% QE (nm) \\
\hline 155 & $\mathrm{SiO}_{2} \& \mathrm{MgF}_{2}$ & 58.1 & 158 & 14 \\
\hline 205 & $\mathrm{SiO}_{2} \& \mathrm{Al}_{2} \mathrm{O}_{3}$ & 89.6 & 205 & 22 \\
\hline $255 \mathrm{a}$ & $\mathrm{SiO}_{2} \& \mathrm{Al}_{2} \mathrm{O}_{3}$ & 85.6 & 254 & 31 \\
\hline $255 \mathrm{~b}$ & $\mathrm{HfO}_{2} \& \mathrm{Al}_{2} \mathrm{O}_{3}$ & 97.8 & 255 & 29 \\
\hline $255 \mathrm{c}$ & $\mathrm{MgF}_{2} \& \mathrm{Al}_{2} \mathrm{O}_{3}$ & 97.7 & 255 & 27 \\
\hline $205 \mathrm{a}$ & $\mathrm{MgF}_{2} \& \mathrm{Al}_{2} \mathrm{O}_{3}$ & 97.2 & 305 & 56 \\
\hline $205 \mathrm{~b}$ & $\mathrm{HfO}_{2} \& \mathrm{Al}_{2} \mathrm{O}_{3}$ & 82.5 & 307 & 45 \\
\hline $205 \mathrm{c}$ & $\mathrm{SiO}_{2} \& \mathrm{Al}_{2} \mathrm{O}_{3}$ & 98.8 & 305 & 42 \\
\hline
\end{tabular}



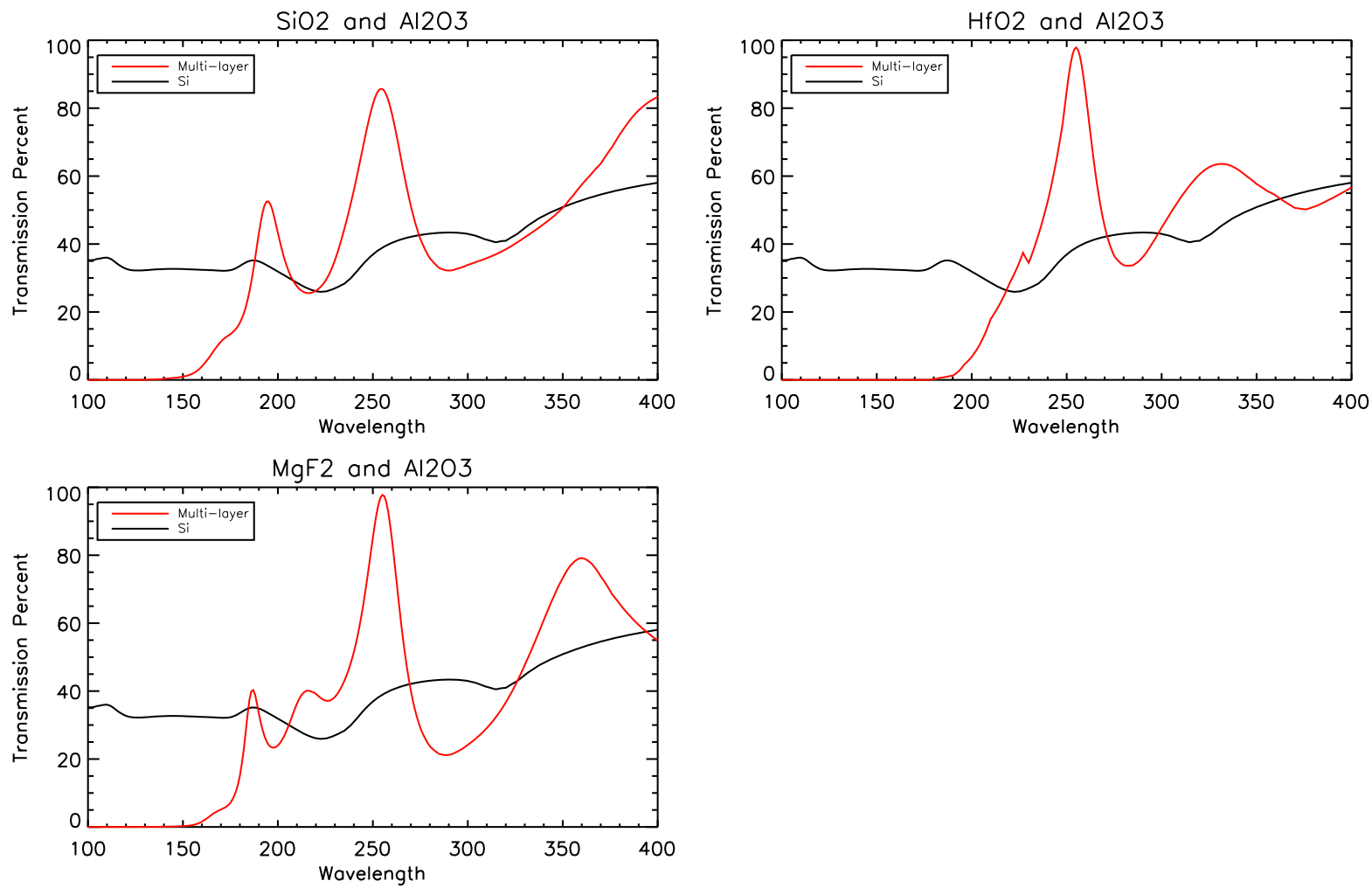

Figure 2. Expected transmission for three possible multilayer models optimized for maximum transmission at $255 \mathrm{~nm}$ using $\mathrm{SiO}_{2}, \mathrm{Al}_{2} \mathrm{O}_{3}, \mathrm{HfO}_{2}$, and $\mathrm{MgF}_{2}$ as materials. Each film uses only two materials, listed above each plot.

on delta-doped CCDs. Previous work on delta-doped CCDs has shown good fidelity to model predictions. Thicknesses were verified in a ellipsometer.

\section{TESTING AND RESULTS}

Tests were conducted at a reflectance set-up at Columbia University. The samples were placed in a vacuum chamber maintained at less than $1 \times 10^{-4}$ torr for the duration of the measurement. An Acton monochrometer fed by a focused deuterium lamp provides light from $120 \mathrm{~nm}$ to $600-700 \mathrm{~nm}$. The light then is then reflected off samples at any angle of incidence from nearly normal $\left(5^{\circ}\right)$ to straight through, in the case of transparent substrates. In testing films grown on $\mathrm{Si}$ substrates, reflectance was at $10^{\circ}$ from normal. For transparent substrates, usually fused silica, the light was able to pass directly through the sample. Current measurements were taken in using a PMT with a scintillator, from McPherson. For each sample the following set of measurements were made: direct emission from the lamp, reflected emission from the sample, reflected emission from a bare Si standard, reflected ambient light not directly in the path of the light. These measurements were then used to calculate direct reflectance from the sample and $\mathrm{Si}$ (as a standard).

Samples measured are as follows:

1. 5-layer film of $\mathrm{Al}_{2} \mathrm{O}_{3}$ and $\mathrm{SiO}_{2}$ optimized at 190nm. See Figure 4.

2. 3-layer film of $\mathrm{Al}_{2} \mathrm{O}_{3}$ and $\mathrm{SiO}_{2}$ optimized at 190nm. See Figure 5 .

\section{DISCUSSION}

We find that films made with ALD are able to match expected values for reflectance and absorption with great accuracy. Both 3 and 5 layer films match the model very closely, without additional absorption which can 

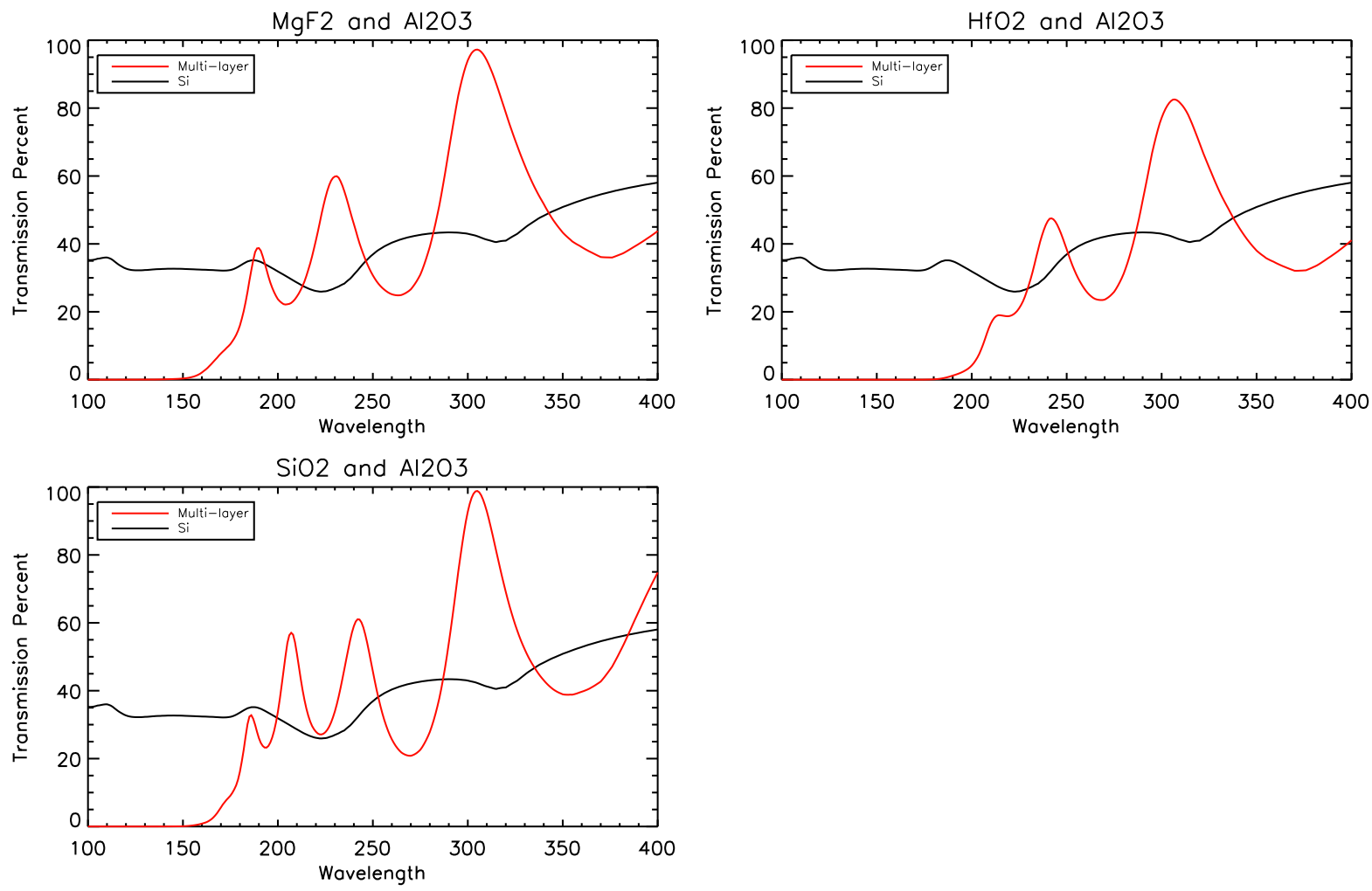

Figure 3. Expected transmission for three possible multilayer models optimized for maximum transmission at 305nm using $\mathrm{SiO}_{2}, \mathrm{Al}_{2} \mathrm{O}_{3}, \mathrm{HfO}_{2}$, and $\mathrm{MgF}_{2}$ as materials. Each film uses only two materials.

indicate interaction between the silicon substrate and coating layers. We are able to achieve greater than $80 \%$ transmission for the 5-layer film, and expect higher QE with more carefully grown layers. This is a greater than $50 \%$ improvement over the measured QE for a single layer film from our previous work.

In all of our films, we had standard errors related to ALD growth. Typically deposition errors result from inaccurate growth curves for new materials (i.e. thickness of growth per cycle), and these errors are easy to correct with accurate characterization. Typical growth curves are also calculated by growing samples on Si test wafers. But in our multi-layers, for example, $\mathrm{SiO}_{2}$ is grown on existing layers of $\mathrm{Al}_{2} \mathrm{O}_{3}$, which appears to modify the growth of $\mathrm{SiO}_{2}$. Still, once characterized, the growth is highly repeatable, and we expect a good ability to hit thickness targets in the future.

Despite these small errors, achieving extremely high QE at UV wavelengths is now possible. Application of these films on live devices will proceed soon, starting with an $\mathrm{SiO}_{2} / \mathrm{Al}_{2} \mathrm{O}_{3}$ multi-layer optimized for 205nm. The bandwidth of such a film can be narrowed by increasing the number of layers in the coating. These types of films (high QE, but narrow bandwidth) have many uses. Laser applications, in particular, do not require a wide band. A spectrograph that operates over a narrow range, or one with many detectors each observing a narrow range, could also use these types of coatings to achieve overall high QE. A further solution for a spectrograph consisting of one detector would be to build a ramped AR-coating such that the correct thickness AR-coating was applied to the correct region where a certain wavelength will be detected. At the blue end of the spectrograph detector, the AR coating would be thin, while at the red end, the coating would be proportionally thicker.

Widening the bandwidth, however, and achieving high QE over many tens of nanometers in the UV, is a challenge that still needs to be worked on. Creative use of ALD to create meta-materials, more careful index matching, and the innovative use of 3-d nano-structures (bumps, pyramids, etc.) or ramps, could all be harnessed to create a wide bandwidth, high $\mathrm{QE}$ detector. 

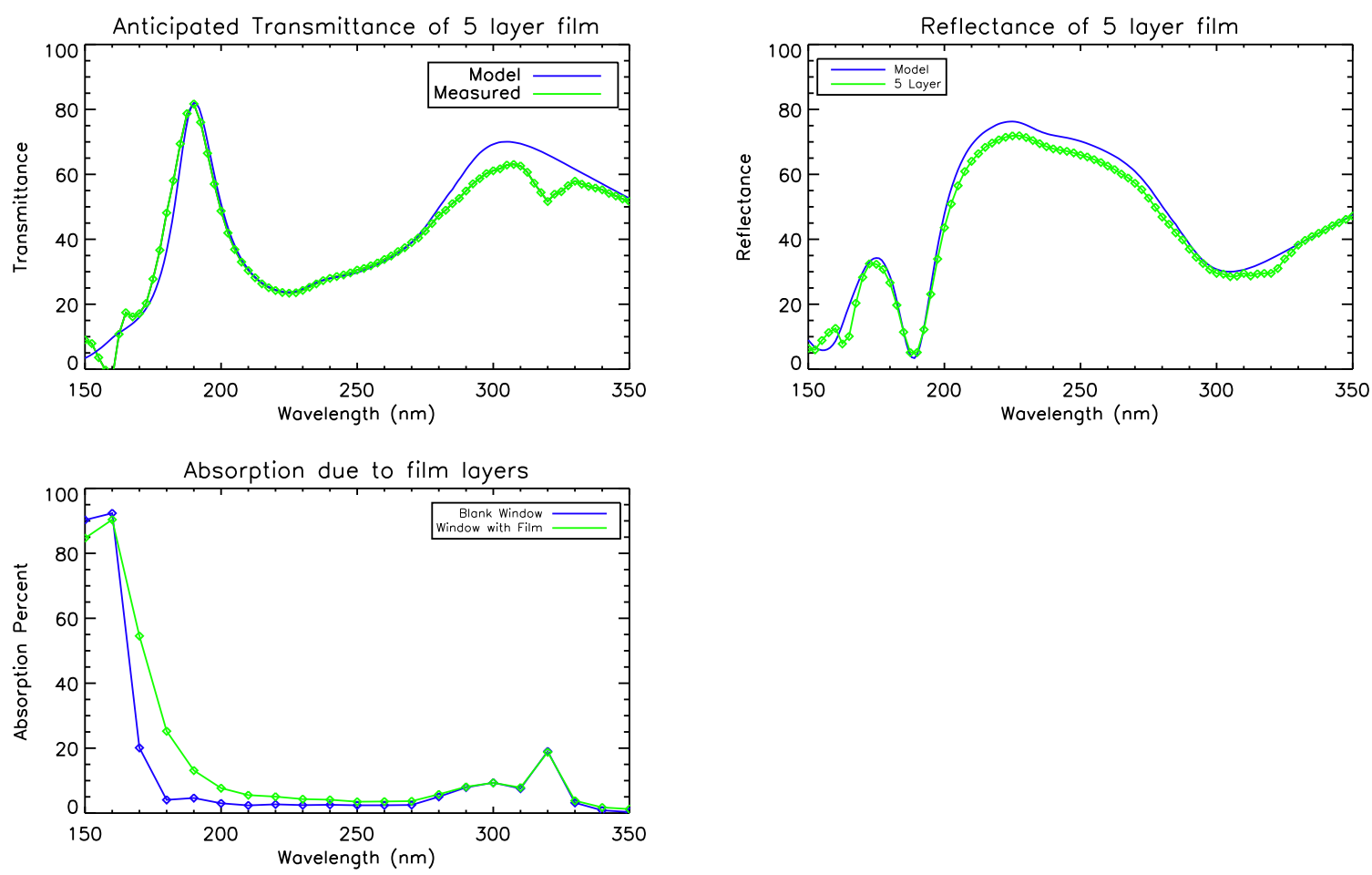

Figure 4. Top Left Estimated transmission for a 5-layer film of $\mathrm{SiO}_{2}$ and $\mathrm{Al}_{2} \mathrm{O}_{3}$. Peak transmission is at $190 \mathrm{~nm}$, and far exceeds previous work at this wavelength (approximately $60 \%$ transmission). This transmission was determined by subtracting the reflectance and absorption from $100 \%$. Top Right Measured reflectance for a 5-layer film of $\mathrm{SiO}_{2}$ and $\mathrm{Al}_{3} \mathrm{O}_{3}$. Minimum reflectance is at 190nm. Bottom Left Measured absorption for 5-layer film on fused silica window. Absorption is due to both the fused silica substrate, as well as the film itself. The film absorption can be measured by the difference between the two lines. Film absorption is rising at shorter wavelengths, but is still minimal at $190 \mathrm{~nm}$ and higher.
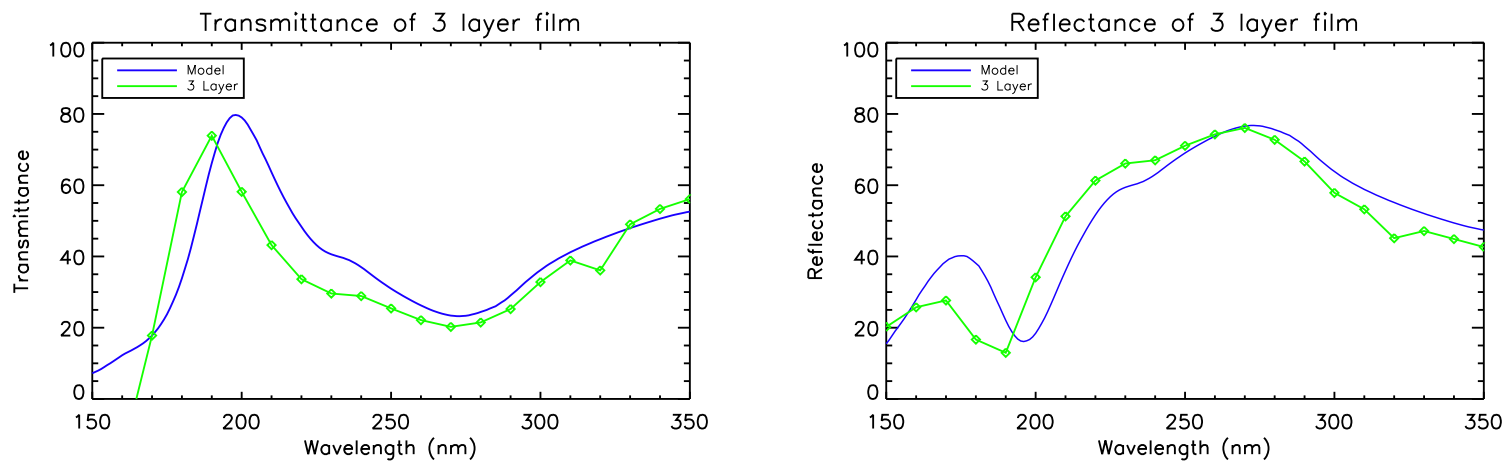

Figure 5. Left Estimated transmission for a 3-layer film of $\mathrm{SiO}_{2}$ and $\mathrm{Al}_{2} \mathrm{O}_{3}$. Peak transmission is at 190nm, slightly below the target of $200 \mathrm{~nm}$. A thinner than expected $\mathrm{SiO}_{2}$ layer is responsible for the discrepancy. The width of the transmittance peak is significantly thicker than that of the peak for a 5-layer film in Figure 4, while the absolute height of the peak is slightly shorter. Right. Measured reflectance for 3-layer film. Minimum reflectance is at $190 \mathrm{~m}$, slightly below the target of $200 \mathrm{~nm}$. 


\section{ACKNOWLEDGMENTS}

The authors wish to thank Mike Lee of JPL for his assistance with ALD processes. The research was carried out in part at the Jet Propulsion Laboratory, California Institute of Technology, under a contract with NASA. This work was partially supported by KISS, the W. M. Keck Institute for Space Studies, and by NASA Headquarters under the NASA Earth and Space Science Fellowship Program, NASA Grant NNX11AO07H, and NASA Grant NNX12AF29G.

\section{REFERENCES}

[1] Nikzad, S., Hoenk, M. E., Grunthaner, P. J., Terhune, R. W., Grunthaner, F. J., Winzenread, R., Fattahi, M. M., Tseng, H.-F., and Lesser, M. P., "Delta-doped CCDs: high QE with long-term stability at UV and visible wavelengths," in [Society of Photo-Optical Instrumentation Engineers (SPIE) Conference Series], Crawford, D. L. and Craine, E. R., eds., Society of Photo-Optical Instrumentation Engineers (SPIE) Conference Series 2198, 907-915 (June 1994).

[2] Hamden, E. T., Greer, F., Hoenk, M. E., Blacksberg, J., Dickie, M. R., Nikzad, S., Martin, D. C., and Schiminovich, D., "Ultraviolet antireflection coatings for use in silicon detector design," Applied Optics 50, 4180-4188 (July 2011).

[3] Nikzad, S., Hoenk, M. E., Greer, F., Jacquot, B., Monacos, S., Jones, T. J., Blacksberg, J., Hamden, E., Schiminovich, D., Martin, C., and Morrissey, P., "Delta-doped electron-multiplied CCD with absolute quantum efficiency over $50 \%$ in the near to far ultraviolet range for single photon counting applications," Applied Optics 51, 365 (Jan. 2011).

[4] MacLeod, H. A., [Thin-Film Optical Filters] (1986).

[5] Park, H., Shin, D., Kang, G., Baek, S., Kim, K., and Padilla, W. J., "Broadband optical antireflection enhancement by integrating antireflective nanoislands with silicon nanoconical-frustum arrays," Advanced Materials 23(48), 5796-5800 (2011).

[6] Brewer, A. W. and Wilson, A. W., "Measurements of solar ultraviolet radiation in the stratosphere," Quarterly Journal of the Royal Meteorological Society 91, 452-461 (Oct. 1965).

[7] Tuttle, S. E., Schiminovich, D., Milliard, B., Grange, R., Martin, D. C., Rahman, S., Deharveng, J., McLean, R., Tajiri, G., and Matuszewski, M., "The FIREBall fiber-fed UV spectrograph," in [Society of Photo-Optical Instrumentation Engineers (SPIE) Conference Series], Presented at the Society of PhotoOptical Instrumentation Engineers (SPIE) Conference 7014 (Aug. 2008).

[8] Palik, E. D., [Handbook of optical constants of solids] (1985).

[9] Palik, E. D., [Handbook of optical constants of solids II] (1991).

[10] Goldstein, D. N., McCormick, J. A., and George, S. M., "Al2O3 Atomic Layer Deposition with Trimethylaluminum and Ozone Studied by in Situ Transmission FTIR Spectroscopy and Quadrupole Mass Spectrometry," Journal of Physical Chemistry 112, 19530-19539 (Nov. 2008).

[11] Hatanpaa, T., Ihanus, J., Kansikas, J., Mutikainen, I., Ritala, M., and Leskel, M., "Properties of [mg2(thd)4] as a precursor for atomic layer deposition of mgo thin films and crystal structures of [mg2(thd)4] and [mg(thd)2(etoh)2]," Chemistry of Materials 11(7), 1846-1852 (1999).

[12] Dingemans, G., Helvoirt, C. V., de Sanden, M. V., and Kessels, W. M., "Plasma-assisted atomic layer deposition of low temperature sio[sub 2]," ECS Transactions 35(4), 191-204 (2011).

[13] Liu, X., Ramanathan, S., Longdergan, A., Srivastava, A., Lee, E., Seidel, T., Barton, J. T., Pang, D., and Gordon, R. G., "ALD of Hafnium Oxide Thin Films from Tetrakis(ethylmethylamino)hafnium and Ozone," Journal of the Electrochemical Society 152, G213-G219 (Jan. 2005). 